\title{
El Programa de la Mujer Agraria en Tabasco: expectativas y resultados
}

\author{
The Woman's Program in the Agrarian Sector in Tabasco: Expectations and Results \\ Nélyda Solana-Villanueva \\ Ángel Galmiche-Tejeda \\ Martín de los Heros-Rondenil
}

Resumen: Los programas de género en el medio rural son aún incipientes en México. El Programa de la Mujer Agraria (PROMUSAG) busca generar empleo para las mujeres de áreas rurales y mejorar sus condiciones de vida. Este artículo da cuenta de la evaluación de expectativas y resultados del PROMUSA G en Tabasco en los años 2004 y 2006. Para determinar estos resultados se realizó una encuesta representativa a las beneficiarias del programa en el estado. Se encontró que el apoyo recibido representó principalmente un ahorro familiar más allá de cambios en sus condiciones de vida, aun cuando mencionaron sentirse satisfechas con los resultados del programa. Se comprobó que estas mujeres muchas veces, por falta de tiempo, ceden la operación de sus proyectos productivos. Finalmente, la escasa cobertura da poco margen a resultados positivos.

Palabras claves: género, desarrollo rural, políticas públicas, programas sociales, mujeres.

Abstract: Gender programs in rural Mexico are still in their initial stages. The Woman's Program in the Agrarian Sector aims to generate employment and improve living conditions for women in Rural Mexico. This article looks at the expectation and results of PROMUSAG program in The State of Tabasco between the years of 2004 and 2006. A representative survey was carried out to evaluate the benefits of this program in the state. It was found that the support given by this program was seen by the women as no more than a type of household saving rather than a significant change in family circumstances. Saying this, the women mentioned that they were satisfied with the program and the results. The study found that many women, due to a lack of time, often stopped operating the projects. Finally, there is a small margin for positive results.

Keywords: gender, rural development, public policy, social programs, women.

\footnotetext{
Nélyda Solana Villanueva, maestra en administración y políticas públicas por el Centro de Investigación y Docencia Económicas (CIDE), México. Estudiante de doctorado en geografía por la Universidad Autónoma de Barcelona, España. Investigadora adjunta en el Colegio de PostgraduadosCampus Tabasco, México. Temas de especialización: género y políticas públicas. Correo electrónico: nsolana@colpos.mx.

Ángel Galmiche Tejeda, doctor en geografía por la Universidad de Durham, ReinoUnido. Profesor-investigadorasociadoenelColegiode PostgraduadosCampus Tabasco, México. Temas de especialización: desarrollo sustentable y subsistencia. Correo electrónico: galmiche@colpos.mx.
}

Martín de Los Heros Rondenil, doctor en ciencias políticas y sociales por la Facultad de Ciencias Políticas y Sociales de la UNAM, México. Profesor-investigador de la Facultad Latinoamericana de Ciencias Sociales (FLACSO) sede México. Temas de especialización: evaluación de políticas públicas. Correo electrónico: mheros@flacso.edu.mx.

Enviado a dictamen: 30 de enero de 2013.

Aprobación: 06 de agosto de 2013.

Revisiones: 2. 


\section{Introducción}

L os últimos resultados sobre medición de la pobreza en México muestran que el $46 \%$ de la población se encuentra en estas condiciones. ${ }^{1}$ La pobreza ha aumentado principalmente en el medio rural mexicano, al pasar del 62.4\% en 2008 al 64.9\% en 2010, y en particular en las zonas indígenas, donde en esos mismos años las personas pobres pasaron del 75.3\% al 77\% (CONEVAL, 2011: 6). El incremento de la pobreza ha impactado profundamente en los hogares campesinos, modificando la configuración del empleo rural, la seguridad alimentaria (Appendini y De Luca, 2006: 4) y las propias condiciones de reproducción social en esos hogares.

Actualmente, el área rural cuenta con 24 millones de habitantes (INEGI, 20lla) y, aunque el gasto social rural del país creció significativamente (Merino, 2009: 6) para combatir la pobreza y la marginación a partir de 1994, las inequidades en estas zonas se han mantenido. Por ejemplo: "la probabilidad de caer en pobreza en los hombres y mujeres indígenas es alrededor de $38 \%$, casi el doble que su contraparte no indígena que ronda el 20\%" (Cordourier-Real, 2010: 64); el riesgo de morir durante el embarazo, el parto o el puerperio ${ }^{2}$ se triplica en los municipios que son mayoritariamente indígenas (Cordourier-Real, 2010: 66) en relación con los municipios que tienen presencia indígena más baja; en ocho regiones indígenas las mujeres reportan que la violencia de la pareja $\mathrm{a}^{3}$ en 2008 estuvo cuatro puntos porcentuales por encima de la prevalencia nacional (INSP, 2008: 119); en 2010, el 17.8\% de la población de estrato socioeconómico muy bajo - tomando en cuenta que se concentran en el medio rural- mencionó que sus derechos no eran respetados por su color de piel, contra el 11.6\% de la población de estrato socioeconómico medio alto/alto (CONAPRED, 2010: 41), entre otras variables. Es decir, en el medio rural subyacen de manera palpable inequidades que se mantienen aún con inversiones en políticas sociales que van más allá del ingreso y que tienen que ver con los procesos de igualdad de oportunidades por condición de etnia, género, clase o sexualidad. Las relaciones estructurales que perpetúan las inequidades son tan abrumadoras que, de no haber avances sustantivos en estas brechas, se acentuarán las complejidades de la exclusión social. ${ }^{4}$

En este contexto, las políticas de género en el medio rural han intentado centrar sus esfuerzos en disminuir las inequidades de estas regiones a través de la promoción del empleo. Este artículo analizó las características de las beneficiarias del Programa de la Mujer Agraria en Tabasco de los años 2004 y 2006, la percepción de los beneficios del programa en sus vidas a través de su opinión antes y durante la operación del programa, si contribuyó a mejorar sus condiciones de vida, los problemas que tuvieron y sus demandas. En 2004 y 2006, este programa pretendía integrar a las mujeres al mercado laboral y, de esta manera, generar ingresos en sus hogares. Los resultados del programa muestran que los proyectos generaron un breve bienestar para estas mujeres, pero no lograron la integración económica que se pretendía ni cambiaron los niveles de desigualdad en los que vivían. Esto se debió principalmente a que no tomaron en cuenta las relaciones de poder inherentes a las relaciones de género en el medio rural y a que la cobertura del programa no es significativa para atender este problema público.

\section{La perspectiva de género y la complejidad rural}

Según Lagarde (1996: 11), el género es la categoría correspondiente al orden sociocultural configurado sobre la base de la sexualidad. A partir de esta categoría se establece una organización genérica donde roles y deberes configuran a los sujetos sociales. Es una "estructura estructurante" (Bourdieu, 1995) que se interrelaciona o crea interseccionalidad (Lugones, 2008: 10) con otras categorías como raza, sexualidad o clase; que construye, reconstruye, transforma y resignifica las identidades y relaciones de acuerdo con la realidad específica en que se vive (Olivera, 2010: 73). Las implicaciones de la teoría de género ponen de relieve principalmente las relaciones estructurales de poder entre hombres y mujeres y la distribución inequitativa de este poder y sus recursos -económicos, sociales, físicos, simbólicos y de identidad-. 
Las mujeres del medio rural conviven en condiciones de desigualdad, lo cual se atribuye principalmente a su posición y condición de género (Lagarde, 1990). La división sexual del trabajolas coloca como reproductoras biológicas, sociales y físicas de la familia, otorgándoles roles de cuidadoras y madres. El trabajo de estas mujeres, considerado exclusivamente femenino, se circunscribe al terreno doméstico y extradoméstico: trabajo en el campo, en el cuidado de los animales de traspatio (Arizpe y Botey, 1986: 143), atención a los miembros de la familia y labores del hogar. Todo esto conlleva una sobrecarga de trabajo que puede implicar hasta tres jornadas laborales al día y que la mayoría de las veces es invisible para el resto de la familia o para la sociedad. ${ }^{5}$

Las condiciones de desigualdad en las zonas rurales han generado y profundizado la sobrecarga de trabajo de las mujeres rurales. Se han incorporado o intentado integrar al mercado laboral extradoméstico como estrategia para disminuir la marginación de sus hogares, lo que ha provocado el aumento de su esfuerzo físico y de su jornada laboral (Arizpe y Botey, 1986: 143; Zapata-Martelo, 2005: 10), además de haber traído consigo cambios en su posición como mujeres en la familia y la comunidad (Appendini y De Luca, 2006: 56), lo cual ha impactado en el sistema de prestigios y estructuras de poder donde conviven estas mujeres (Martínez, 2005: 242). Los hogares son los espacios donde se recrean las relaciones de poder y autoridad (Kabeer, 1998; Tepichin, 2011: 14) y donde se determinan las condiciones sociales y de trabajo para sus integrantes (Tepichin, 2011: 15). Los cambios empujan a las mujeres a trabajar más o a participar en otras actividades que pueden moverlas a situaciones de conflicto o de sincretud de género (Lagarde, 1996), dejándolas en condiciones de mayor inequidad si estos cambios no se realizan a través de procesos de transformación de poder personal y manejo de conflictos: "se empuja a las mujeres a un círculo de cambio sin transformación" (Tepichin, 2010: 33). La inclusión de las mujeres rurales en actividades extradomésticas puede transgredir las costumbres familiares y comunitarias y, como señala Olivera (2010:
82), acumularse en forma de culpa, lo cual implica costos sociales y emocionales altos (Martínez, 2005: 242).

Estos procesos han ampliado las condiciones de inequidad de las mujeres rurales, indígenas y campesinas, y las han lanzado a procesos donde se refuerza su condición subordinada: "modernizando su rol se le incluye además la obligación de ser una esforzada trabajadora, ya sea en la parcela familiar o fuera de casa, a fin de conseguir los recursos económicos para completar lo necesario para el sostenimiento familiar" (Olivera, 2010: 77). El subsidio del trabajo de las mujeres es parte fundamental en los ingresos de los hogares rurales y ha permitido que no sean más pobres de lo que son ahora.

En las transformaciones históricas de la sociedad rural emerge una nueva ruralidad que muestra que la agricultura no es la principal fuente de empleo e ingreso ni de supervivencia del hogar, una mayor movilidad de personas, bienes e información, y un mayor reconocimiento de la vinculación entre lo global y local (Appendini y Torres, 2008; Carton de Grammont, 2004). Estos factores transforman a los actores incluidos en estos espacios. En estos nuevos modos de vida, donde no necesariamente ser del medio rural significa ser campesino, las mujeres se circunscriben y, como mencionan Appendini y Torres (2008: 19), en tanto receptoras de programas asistenciales, las mujeres se han convertido en mediadoras de la nueva intervención estatal.

\section{Política pública de género y el medio rural}

Las políticas públicas de género ${ }^{6}$ en México en los últimos 12 años (INMUJERES, 2008) han cobrado gran importancia dentro del discurso y la gestión gubernamental. Los antecedentes de estos programas se remontan a los años cincuenta (Tepichin, 2010: 25) con propuestas sobre cuidado infantil y la maternidad sin un claro énfasis en los problemas de las mujeres. A partir de los años ochenta, se establecieron de forma más consistente estrategias de política pública de género en el país que han transitado en tres tendencias 
desde el análisis feminista: 1) Mujeres en el desarrollo (MED), donde a las mujeres se les reconoce como parte del desarrollo, pero son vistas como consumidoras de servicios, usuarias de recursos, sujetas pasivas y agentes económicos (Zapata-Martelo, 2005: 12; Portocarrero y Ruiz, 1990: 28). Estas estrategias enfatizan los roles de madre y cuidadora del hogar, cobijados en los enfoques de bienestar y antipobreza (Zapata-Martelo, 2005: 13);2) Género en el desarrollo (GED), que incorporó la discusión del poder y la desigualdad de género en las estrategias de política pública (Tepichin, 2005: 52) e incluye prácticas que buscan generar avances económicos, sociales y de empoderamiento de las mujeres (Zapata-Martelo, 2005: 19), además de colocar los intereses estratégicos de género en la agenda pública (Kabeer, 1998: 108); y 3) la transversalización e institucionalización de la perspectiva de género, que intenta incorporar la perspectiva de género en todas las acciones de gobierno (Tepichin, 2005: 61) y del que se desprenden políticas para el tratamiento igualitario, las acciones positivas y la promoción de instancias gubernamentales que se responsabilicen de la disminución de las desigualdades sociales de género (Tepichin, 2005: 64).

Las políticas de MED han sido las másimplementadas en el país, son menos complejas y no discuten los procesos de poder inherentes a las estructuras sociales que pueden incomodar a los tomadores de decisiones (Tepichin, 2005: 56). Han incorporado un discurso democratizador, de empoderamiento y de equidad social (García, 2010: 210) que las hace atractivas, aun cuando algunas no las integren en su diseño, operación y evaluación. Esto ha implicado que los esfuerzos públicos-programáticos se enfoquen principalmente en la generación de empleo y alivio de la pobreza (Molyneux, 2006: 2), con procesos de desarrollo social que miran a las mujeres como detonadoras y sostenedoras de procesos de cuidado y cambio comunitario. Además, como señala Moser (1989), hay que reconocer que en la vida cotidiana de las mujeres existen necesidades prácticas de género - condiciones materiales y de control de recursos inmediatos-, y necesidades estratégicas de género - transformación de la ubicación social, económica y política de mujeres y hombres para disminuir la desigualdad - que buscan ser resueltas y que tienen que empatar o competir para ser prioridades en su solución. Por lo anterior, la atención se ha puesto sobre las necesidades que han parecido urgentes o más demandadas, o donde su implementación pueda deparar mayor ganancia política para las agencias públicas.

Las políticas públicas federales de género destinadas al medio rural se han ubicado principalmente dentro de las estrategias cobijadas en los enfoques de bienestar y antipobreza: aquellas que retoman a las mujeres rurales como grupo prioritario de manera explícita, entre éstos el Programa de la Mujer Agraria (PROMUSAG); las que focalizan a población abierta por sus condiciones productivas y de pobreza, como es el caso del Programa Oportunidades; y las políticas que plantean transversalizar el enfoque de equidad en las políticas del sector rural, como fue el caso del Programa Mujeres en Desarrollo Rural y, recientemente, el Programa Nacional para la Igualdad entre Mujeres y Hombres 2008-2012 (INMUJERES, 2008). Todas estas políticas se enmarcan en la promoción del cuidado del hogar y del autoempleo de las mujeres rurales con la consiguiente generación de ingresos para sus hogares. Mantiene una visión instrumental de las mujeres, donde son vistas como agentes de cambios, con cargas de trabajo y responsabilidades extra, y donde la discusión de las inequidades sólo se enfatiza en el terreno monetario.

Los resultados de estas políticas en el medio rural muestran que la incorporación de las mujeres a estos programas no necesariamente se traduce en mejoras en su situación económica o su posición de poder (Tepichin, 2011: 32), y que, si son favorables, algunos cambios duran poco tiempo (Olivera, 2010: 87) porque son poco sustentables al no afrontar las inequidades en la división del trabajo y en el poder en el hogar. Jenson (2009: 27) recomienda tener cuidado con estas políticas destinadas a disminuir las inequidades de género ya que hay una "pérdida" en la traducción de la igualdad — que promueve el feminismo - y la conciencia de género que influye en las perspectivas de la inversión social en las políticas sociales. 


\section{Programa de la Mujer Agraria en Tabasco}

En Tabasco, en 2010 la población en el área rural era de 962599 habitantes, el 43\% de la población total del estado según INEGI (2011a). Dentro de esta población, las mujeres representaban el 49.9\%. Durante el segundo trimestre de 2011, la población ocupada de mujeres en Tabasco que laboraba en actividades agrícolas, ganaderas, silvícolas, y de caza y pesca representó el 0.92\% del total de mujeres ocupadas del estado, un total de 290000 mujeres trabajadoras según la Encuesta Nacional de Ocupación y Empleo (INEGI, 2011b).

En 2007, la proporción de mujeres tabasqueñas que eran consideradas productoras ${ }^{7}$ abarcaba el $22.9 \%$ del total de productores en Tabasco, con un número de 20704 (INEGI, 2008a). Asimismo, las mujeres tabasqueñas que eran ejidatarias y comuneras fueron 22 996, pero sólo representaron el 22\% de las tenedoras de derechos en estos núcleos agrarios (INEGI, 2008b).

En este contextoestatal seimplementael PROMUSAG a través de la Secretaría de la Reforma Agraria (SRA). Esta política federal está destinada a mujeres que habitan núcleos agrarios y opera desde 2003. Se alinea al eje 2, "economía competitiva y generadora de empleos", y al eje 3, "igualdad de oportunidades," del Plan Nacional de Desarrollo (PND) 2007-2012, y al eje 5, "atención a grupos prioritarios", del Programa para el Desarrollo Sectorial Agrario 2007-2012 ${ }^{8}$ (Diario Oficial de la Federación, 2010). Según las últimas reglas de operación 2011, su principal objetivo es:

Contribuir a la generación de empleo e ingreso y mejoramiento de las condiciones de vida de las mujeres de 18 años y más que habitan en Núcleos Agrarios, mediante el otorgamiento de apoyos para la implementación y puesta en marcha de proyectos productivos en sus localidades o para garantías líquidas.

Este objetivo ha variado desde 2003. En un principio se pretendía la integración económica y productiva de las mujeres agrarias, pero en 2011 se enfoca en la mejora del ingreso y en promover su empleo.
El programa lleva operando nueve años y se ha evaluado institucionalmente en siete ocasiones a nivel nacional. La evaluación institucional del programa de 2011 (SRA, 2011) no hizo referencia alguna a la mejora en las condiciones de equidad de las mujeres beneficiarias; referencias a las relaciones de género sólo se mencionan como parte de la operación de los proyectos y se enfocaba en medir la generación de ingreso: se detectó un $16.4 \%$ de incremento en el ingreso mensual promedio de las beneficiadas, compuesto por utilidades y salarios derivados del proyecto. Sólo el 39.2\% de los grupos tuvo utilidades positivas en su primer año. Sin embargo, el Índice de Desempeño de Programas Federales de 2012 (GESOC, 2012) señala que el PROMUSAG es un programa que muestra claramente la dispersión de esfuerzos gubernamentales debido a su bajo nivel de cobertura. Es más, este programa tuvo el más bajo nivel de cobertura $(1.02 \%)$ de la población potencialmente beneficiaria en dicho análisis y, como mencionan: "no tiene posibilidad alguna de resolver significativamente el problema público que le dio origen."

En Tabasco, el programa creció presupuestalmente un 52\% -en términos nominales - hasta 2009. El municipio con mayor importancia en la distribución presupuestal fue Huimanguillo porque en ese momento tenía el más alto índice de marginación en el estado (0.0375) según la CONAPO (2006). El porcentaje de la población atendida fue, en 2004, de apenas el $0.02 \%$ de la población potencial, y del $0.05 \%$ en $2006 .{ }^{9}$ Esto de entrada limita el alcance e impacto del programa ya que su cobertura no alcanzó ni el 1\% de su población potencial.

\section{Materiales y métodos}

Debido a que se quería conocer de manera representativa la percepción y las características de las beneficiarias del programa en el estado, se decidió aplicar una serie de preguntas a una muestra aleatoria en todo el estado. La población de beneficiarias del programa fue de 449 en 2004 y de 1009 en 2006. Para la investigación se aplicó un cuestionario a una muestra aleatoria simple $e^{10}$ de mujeres beneficiarias del PROMUSAG de los años $2004 \mathrm{y}$ 2006 en el estado de Tabasco, con un nivel de confianza 
del 90\% y un margen de error máximo aceptable del 5\%. El tamaño de la muestra propuesto fue de 80 casos para 2004 y 89 para 2006; además, se consideró una tasa de no respuesta del $20 \%$, por lo que se adiciona a la muestra un reemplazo en esa proporción. Fueron aplicados en total 151 cuestionarios a mujeres beneficiadas: 74 correspondieron a beneficiarias en 2004 y 77 en 2006. ${ }^{11}$ Se entrevistó al menos a una integrante de cada uno de los 42 grupos de 2004 y de los 67 grupos de 2006. Esto implicó visitar 15 municipios del estado: Balancán, Cárdenas, Comalcalco, Cunduacán, Centro, Centla, Nacajuca, Jalpa de Méndez, Jalapa, Jonuta, Tacotalpa, Teapa, Tenosique, Macuspana y Huimanguillo. El trabajo de campo se desarrolló desde octubre de 2007 hasta abril de 2008. El análisis estadístico que se utilizó en la investigación fue descriptivo y de contingencia para variables categóricas.

Las percepciones de las usuarias sobre el programa se analizan en tres momentos del proyecto: sus expectativas previas a la operación del programa, durante la implementación y su opinión sobre el resultado final. Las variables utilizadas para interpretar las percepciones de las beneficiarias fueron cualitativas nominales, categóricas y, en algunos casos, opiniones abiertas.

\section{Resultados: características socioeconómicas de las mujeres rurales de Tabasco beneficiarias del PROMUSAG 2004 y 2006}

En 2004, el programa apoyó a mujeres con edades comprendidas entre los 22 y los 65 años. En promedio tenían 41 años cumplidos (ver cuadro 1) y la cuarta parte de las beneficiarias tenía 30 años o menos. Estas mujeres tienen dos años más que el promedio de edad de las beneficiarias nacionales de 2006 (SRA, 2008: 19). Si bien se mantiene la edad promedio para las beneficiarias de 2006 con respecto a las de 2004, se amplía el rango de edad de 43 a 58 años, de las que aproximadamente el $4 \%$ tiene más de 65 años. Ésta era una población relativamente joven, que en promedio tenía un horizonte de participación en la actividad laboral de 25 años más.
Otra variable importante sobre las beneficiarias fue el nivel educativo. Los resultados muestran que más de la mitad de esta población terminó la primaria: 58\% y 56\% en 2004 y 2006 respectivamente. Estos datos son parecidos a la escolaridad media de las beneficiarias nacionales apoyadas en 2006, que fue de 6.5 años (SRA, 2008: 19). Es decir, estas mujeres sólo alcanzaron a terminar la primaria y el primer año de secundaria. Para tener un mejor acercamiento a las condiciones de educación de las beneficiarias, se revisaron dos variables relacionadas con habilidades "saber leer y escribir" y "sacar cuentas". Los resultados muestran que, de cada diez mujeres, ocho saben leer y escribir y "sacar cuentas". Estos hallazgos nos dan una idea de los niveles de instrucción de las beneficiarias, los cuales impactarán en la toma de decisiones, en el uso de los recursos del programa, en la capacidad de innovación y en el manejo administrativo de los proyectos. Asimismo, el grado de escolaridad de las beneficiarias podría constituir un factor limitante para el programa, por lo que se requiere un continuo apoyo en los temas concernientes a la administración y gestión de los proyectos.

Respecto al estado civil de las beneficiarias, los resultados muestran que la mayoría - $74 \%$ y $86 \%$ tenían pareja. Estas cifras son similares a los datos reportados para las beneficiaras nacionales de 2006 (SRA, 2008: 19), donde el 73.9\% reportó estar casada o vivir en unión libre. Se recopiló información del ingreso total promedio de los hogares. ${ }^{12}$ El ingreso - a precios nominales de 2008- fue de 4199 pesos para las beneficiarias en 2004 y de 4528 pesos para las beneficiarias en 2006. Por lo que respecta al ingreso por trabajo de estas beneficiarias, ${ }^{13}$ su contribución al ingreso total del hogar en ambos años representa el 44\% y $46 \%$, respectivamente, tal como se puede observar en el gráfico 1.

El ingreso mensual per cápita de los hogares de las beneficiarias de 2004 fue de 1000 pesos y de 1063 pesos para las beneficiarias de 2006. Con este nivel de ingreso, en 2008 se encontraban debajo de la línea de bienestar en el medio rural, ${ }^{14}$ pero aún arriba de la línea de bienestar mínimo de ese año en el medio rural reportado por CONEVAL (2011). En contraparte, el ingreso promedio 
mensual de las beneficiarias nacionales en 2008 (SRA, 2008: 122) fue de 739.49 pesos por debajo del ingreso mensual per cápita reportado para las beneficiarias de Tabasco en este estudio.

Sobre su condición como ejidatarias o avecindadas, sólo el 20.4\% y el 15.3\% señalaron en ambos años pertenecer a este tipo de productoras. El porcentaje reportado como ejidatarias para las beneficiarias nacionales de 2006 (SRA, 2008: 79) fue del 16.93\%, así que los datos estatales están cercanos a la media nacional.

El promedio de tiempo que dedican las beneficiarias de 2004 y 2006 al trabajo en el hogar es de entre una y dos horas más que las nueve horas diarias reportadas a nivel nacional en el 2009 por el INMUJERES (2010: 6). Este dato es importante porque impacta en la disponibilidad de tiempo para incorporarse a otras actividades extradomésticas y recalca la importancia del espacio doméstico en la vida de las beneficiarias.

Todas estas características señalan a una población estatal de mujeres rurales beneficiadas con características muy similares a la población nacional de mujeres beneficiarias en 2006 reportada por las evaluaciones de la Secretaría.

\section{La operación del PROMUSAG de 2004 y 2006 en Tabasco}

En 2004, el PROMUSAG apoyó a 449 mujeres que estaban incluidas en 47 grupos destinados principalmente a proyectos de bovinos doble propósito (48.9\%) y bovinos de cría y engorda (21.2\%). El programa distribuyó los beneficios a 12 municipios, especialmente a los municipios de Huimanguillo y Nacajuca que concentraron el 46.8\% de los grupos. En el año $2006 \mathrm{el}$ número de grupos apoyados se triplicó a 106 e involucró a 1009 mujeres beneficiadas. De estos grupos, el 57.5\% se dedicaron a proyectos de bovinos doble propósito, el $13.2 \%$ a la cría y engorda de porcinos y el $9.4 \%$ a proyectos de bovinos de cría y engorda principalmente. $\mathrm{Al}$ igual que en años anteriores, se beneficiaron 12 municipios, pero tres concentraron el 51.8\% de los apoyos: Huimanguillo, Cárdenas y Nacajuca.
El 64\% de las beneficiadas en 2004 y el 42\% de las beneficiarias en 2006 eligieron el tipo de proyecto porque lo consideraron como una actividad sencilla o fácil. El 80\% y 90\% de las beneficiadas, en los dos años respectivamente, realizaron las actividades planeadas inicialmente en el proyecto, lo cual indica que los grupos organizaron mínimamente sus actividades y cumplieron con el plan. Las beneficiarias que reportaron que no recibieron información del programa en tiempo y forma fueron el 23\% en el 2004 y el 35\% en el 2006 (ver cuadro 2).

Tanto en 2004 como en 2006, casi una tercera parte de las beneficiarias - 37\% y $32 \%$, respectivamenteoptó por trabajar el proyecto individualmente, es decir, abandonar el grupo. Esto es indicativo de una cultura individualista, originada por falta de confianza en el trabajo grupal. Al respecto, Galmiche-Tejeda y Townsend (2006) reportan que, en general, los grupos productivos de hombres y mujeres en Tabasco tienden a fracasar principalmente por conflictos sobre las cargas de trabajo y la repartición de beneficios, y que los grupos más estables son aquellos compuestos por familiares. Es por esto que, como se menciona más adelante, la confianza entre las integrantes es esencial para el manejo exitoso del proyecto.

Asimismo, la mitad de las beneficiarias de 2004 y una tercera parte de las beneficiadas de 2006 operaron personalmente el proyecto. El resto de ellas contrató a alguien o dejó a otro miembro de la familia la responsabilidad de las actividades. Esto tuvo implicaciones para el programa ya que, si los objetivos eran generar empleo e ingresos para las beneficiarias, esto no se logró porque ellas delegaban el empleo y las responsabilidades a otras personas. Las razones sociales que explican esta práctica están vinculadas, en primer lugar, a la estricta división sexual del trabajo en las áreas rurales. La mayoría de los proyectos desarrollados por los grupos de mujeres fue con ganado bovino, actividad que tradicionalmente sólo incluye a mujeres cuando no hay familiares varones que la realicen (Arizpe y Botey, 1986:148). Aunque en muchas zonas del mundo la ganadería (Maeda-Machang'u et al., 2000) y la agricultura (Dixon, 1982; Mink, 1993) son parte de las labores femeninas, en Tabasco las actividades 
agropecuarias, al desarrollarse fuera del hogar, son consideradas masculinas, por lo que, como lo señalan las beneficiarias, las delegaron a los varones de la familia, quienes tienen experiencia previa y a quienes no se les verá mal trabajando en este ámbito.

La segunda razón es el tiempo del que disponen las mujeres rurales. En promedio, las beneficiarias de los dos años destinaban entre 10 y 11 horas del día a labores del hogar, así que no tenían mucho tiempo para operar los proyectos. De las mujeres que sí trabajaron personalmente los proyectos (ver cuadro 2), el 46\% destinaba entre 11 y 18 horas a sus labores del hogar, por lo que cumplían probablemente con dobles jornadas laborales.

En ambos años, más de la tercera parte de las beneficiarias señaló que nunca recibió asesoría técnica ni capacitación sobre el proyecto. Este tema es de gran importancia ya que, como señalan Pérez y otros autores (2009), se requiere de acompañamiento para sostener los procesos de operación de los proyectos y para además apoyar los cambios que estas mujeres sufrirán, tanto a nivel personal como familiar, por incursionar en trabajos extradomésticos no tradicionales. La adecuada capacitación para la innovación es un elemento esencial para el éxito de un proyecto productivo (Reij y WatersBayer, 2001), y más en una población con baja escolaridad pero con gran experiencia práctica en actividades rurales. Si consideramos que estos proyectos, además de promover la incursión de las mujeres en actividades no tradicionales, también buscan empoderarlas, entonces la capacitación se convierte en una piedra angular para la continuidad de los proyectos y así lograr un cambio cultural sostenido.

Entre los objetivos de la política social se encuentra que los programas y proyectos sean sostenibles. Una variable que permite acercarnos a ese objetivo es la sobrevivencia de los grupos en este programa. Los resultados muestran que, si bien existe una "mortandad" relativamente importante de los grupos, principalmente el de beneficiarias de 2004 (35\%), el porcentaje de sobrevivencia de los grupos dos años después sigue siendo importante - el 64.5\% de los grupos formados en 2004 y el 69\% de aquellos formados en 2006-. ${ }^{15}$
Estos datos son similares a los reportados por la tasa de sobrevivencia de las beneficiarias nacionales de 2006, que alcanzó el 66.27\% (SRA, 2008: 103). Esta evaluación señala que la duración de los grupos y de los proyectos depende fundamentalmente de cómo se originó el grupo y de la capacidad de organización que mantienen internamente; de ahí que la mayoría de las beneficiarias en ambos años señaló que "el trabajo unido", la confianza en el grupo, es el factor principal de éxito en los proyectos, como igualmente lo señalan Cañada y Zapata-Martelo (2005: 55).

De esta manera, algunos aspectos del programa fueron adaptados por las beneficiarias a sus necesidades personales. Los cambios que efectuaron en la operación impactaron los fines formales del programa dado que no se generó empleo directo entre las participantes, además de que en términos de focalización sólo se tuvo una cobertura menor al $1 \%$ de la población potencial en el estado.

\section{Percepción de cambios en su vida por parte de las beneficiarias del PROMUSAG 2004 y 2006}

El PROMUSAG despertó altas expectativas entre las beneficiarias antes de comenzar a operar (ver cuadro 3). Las beneficiarias consideraban que la implantación de este programa les ayudaría a salir de la pobreza. Dicha expectativa fue mayor entre las beneficiarias de 2004 (85\%). La disminución en 2006 podría haber estado influida por los resultados de años anteriores dado que el programa opera en las mismas comunidades y municipios, aunque con otros grupos y personas.

La mayoría de las beneficiarias de 2004 y 2006 (69\% y $79 \%$ ) se sintió satisfecha con los beneficios recibidos, principalmente porque el proyecto les representó una forma de "ahorro" al que podían echar mano cuando tuvieran algún problema, ya sea de salud, educación o alimentación, como se observa en el cuadro 3. De este modo, se puede observar que la satisfacción es mayor cuando se tienen expectativas realistas de los beneficios de un programa. Los resultados presentados coinciden con la evaluación nacional del programa en 2008 (SRA, 2008: 94), donde se determinó que el 73.24\% 
de las participantes estaba satisfecho con los beneficios obtenidos con el proyecto.

Al preguntarles de manera retrospectiva qué esperaban del programa en cuanto a ayudarlas a vivir mejor y si sus expectativas se cumplieron, la mayoría de las beneficiarias en ambos años respondió que "poco" (ver cuadro 3). Desde un inicio esperaban un cambio mínimo en su vida por el proyecto, y ésta fue precisamente la percepción final del resultado que obtuvieron. Al revisar el tema de la pobreza, más del 50\% (ver gráfico 2) manifestó que el programa les ayudaría a salir de ella, y además señalaron que el apoyo otorgado cambió su vida. Estos resultados son interesantes porque, aunque la mayoría de las mujeres beneficiarias mencionó que no esperaban que el programa las ayudara a vivir mejor, sí resultó un apoyo una vez operado. Aunque las respuestas parecen contradecirse, esta divergencia se podría explicar porque, cuando se incorporaron al programa, las beneficiarias conocían las limitaciones de actuación y tiempo que supondría el proyecto, así que intuían que probablemente éste no resolvería sus necesidades económicas totales. El proyecto serviría como medio para adquirir un "patrimonio" del cual echar mano ante emergencias. Éste es precisamente el aspecto que más valoraron. Los semovientes son vistos por los habitantes rurales como bienes que pueden capitalizar al hogar (Rota, 2010: 12), y que con un buen manejo pueden reproducirse y aumentar el hato familiar. Precisamente, las respuestas dadas por las beneficiarias en 2004 señalan que ser parte del programa fue positivo porque les representó un ahorro del que podían echar mano para solucionar problemas de enfermedad o gastos en educación (17 de 61 menciones):

Cuando estuve enferma vendí la primera cría de la becerra. La becerra que quedó es una ayuda, ya sea para una enfermedad o emergencia (beneficiaria, proyecto ganado doble propósito, Centla, Tabasco). Vendí el ganado antes de las inundaciones. Vendí cuatro cabezas, fueron 12000 pesos. Ese dinero lo tengo ahorrado en el banco, me sentía más responsable [cuando trabajaba en su proyecto], era trabajo extra (beneficiaria, proyecto ganado bovino, Nacajuca, Tabasco).

Me permitió comprar bienes diarios para vivir. Antes tenía que viajar distancias. Tener la vaca es un ahorro, puedo lograr mantener a mi familia, sacar adelante a mis hijos (beneficiaria, proyecto tienda de abasto, Tacotalpa, Tabasco).

Antes no tenía y ahora siento que tengo un ahorro. Los animales se han reproducido, es un legado para los hijos, para dejarle algo a los hijos (beneficiaria, proyecto ganado bovino, Macuspana, Tabasco).

Del mismo modo, las beneficiarias del año 2006 detallaron que el beneficio obtenido en el programa les representó "una esperanza" o "mejor futuro" (nueve menciones de 44 respuestas):

El ganado se va reproduciendo y eso me da esperanza de que a futuro pueda echar mano de un animal (beneficiaria, proyecto ganado bovino, Jonuta, Tabasco).

Me daba la oportunidad de ocupar mi tiempo y no estar metida en casa. Además, el alimento representó una fuente de ahorro (beneficiaria, proyecto de porcinos, Centro, Tabasco).

Sí cambió mi vida. Me gusta trabajar en el proyecto, puedo salir adelante con mis hijos, me relaciono más con las otras personas, me despejo la mente (beneficiaria, proyecto agroindustrial, Teapa, Tabasco).

Sin embargo, también existieron percepciones negativas del programa. Las beneficiarias de 2004 reportaron el mayor número de respuestas negativas (ocho de 61 menciones), principalmente porque el proyecto implicó demanda de tiempo y esfuerzo, sin beneficios claros:

El cambio fue negativo, me hizo trabajar más, no podía salir, dejé descuidada mi parcela (beneficiaria, proyecto de porcinos, Comalcalco, Tabasco).

Me las vi duro, era mucho trabajo iacarrear agua! 
Los vendí como alimento (beneficiarias, proyecto de porcinos, Nacajuca, Tabasco).

Ya no seguí, me retiré porque mi niño se enfermaba. Ya no tuve tiempo, avisé que no continuaba (beneficiaria, proyecto taller de costura, Nacajuca, Tabasco).

Los cambios negativos expresados por las beneficiarias de 2006 (cuatro menciones de 44 respuestas) fueron principalmentela pérdida de ganado en las inundaciones de 2007:

Porque las esperanzas que tenía de ahorrar se terminaron al morir el ganado en la inundación (beneficiaria, proyecto de bovinos, Centro, Tabasco).

\section{Discusión}

Los cambios en la vida de estas mujeres se dieron porque el programa abordó, aunque de manera muy incipiente, las necesidades prácticas de las beneficiarias dotándolas de un bien material que pudo resolver un problema cotidiano. Sin embargo, de ninguna manera el programa modificó la división sexual de trabajo y la distribución de recursos en la comunidad y familia, como lo señala Tepichin (2005: 56). Más aún, no hay evidencia de que el programa movió a la familia de las beneficiarias a menores niveles de pobreza. La evidencia de otros programas o estrategias (Olivera, 2010: 87) que incorporan a las mujeres rurales en proyectos de este tipo apunta a que son favorables por poco tiempo y no resuelven sus necesidades económicas porque las beneficiarias no pueden dedicar todo su tiempo a estas actividades y porque la gestión real de estos proyectos es compleja y de difícil manejo para las mujeres rurales - por ejemplo, administración, producción o capacitación-. Notamos que las mujeres rurales son empujadas a negociar con una agenda de gobierno que coadyuva parcialmente a resolver sus necesidades a mediano plazo, para entrar a las nuevas prácticas con desventajas estructurales que pueden tener más costos que beneficios.
Los problemas mencionados dejan ver que las complicaciones en el manejo del tiempo y la sobrecarga de trabajo son resultados adversos de su participación en este programa. La ampliación de la jornada laboral en tiempo y esfuerzo para las mujeres trabajadoras ha sido un tema bastante estudiado en el mundo (Benería y Sen, 1981) y en Tabasco (Tuñón et al., 2007). Se conoce que la entrada de las mujeres en el mercado laboral implicó, más que un cambio de actividad, una duplicación de trabajo (Torres, 1989). El tema fue abordado en Tabasco por Galmiche y Townsend (2006) con mujeres acuicultoras, quienes afirmaron que los programas que buscan mejorar las condiciones de las mujeres se enfocan principalmente en la generación de ingreso, pero ignoran los costos sociales en sus vidas, que pueden incrementar la inequidad.

Aparentemente, esta oferta de políticas es menos compleja porque su lógica multiplicadora parte de que la generación de empleo disminuirá las brechas de inequidad, pero al no percatarse del conjunto de jerarquías sociales y de las nuevas dinámicas rurales, limita sus alcances y efectividad. Por ello, el diseño de políticas públicas debería adoptar una perspectiva sistémica que permita identificar los factores que interfieren en que los objetivos originales se cumplan y llevar a cabo acciones para reducir su efecto. En este sentido, los programas productivos deberían ir acompañados de acciones educativas para distribuir la carga doméstica entre todos los miembros del hogar. No se puede alcanzar un verdadero desarrollo humano si la generación de ingreso de las mujeres se realiza a expensas del aumento de su propia vulnerabilidad.

\section{Conclusión}

El programa ha beneficiado a las mujeres en sus necesidades inmediatas, pero ha significado un aumento en sus cargas de trabajo y responsabilidades de organización que muchas veces no pueden sostenerse en el hogar. Su oferta se dirige a las actividades económicas tradicionalmente asignadas a las mujeres, particularmente las del medio rural —como economía de traspatio o artesanías-, sin abordar seriamente la 
problemática desde una perspectiva teórica consistente y actualizada. Para poder planear y diseñar políticas públicas de género en el medio rural, se requiere integrar las necesidades prácticas y estratégicas de género, como señala Tepichin, de manera tal que se transformen las condiciones de inequidad dentro de los hogares y en la comunidad. Se debe atender apropiadamente el tema de la capacitación y el tipo de agrupación que funciona para estos proyectos. El que sea operado por terceros o individualmente pone de relieve qué forma de agrupaciones se están promoviendo. Los grupos entre parientes podrían ser una alternativa para el trabajo colectivo.

La división sexual del trabajo es un factor determinante en el resultado de los proyectos productivos desarrollados por las mujeres. Éste es un tema que, en el caso del PROMUSAG, no se considera en el diseño y análisis de los proyectos productivos. La literatura disponible indica que las mujeres desarrollan mejor actividades que se dan cerca del espacio doméstico. Cambiar este rasgo cultural en pos del empoderamiento debe evaluarse considerando acciones que no provoquen conflictos en el hogar ni en la comunidad. El hecho de que las mujeres opten por proyectos agropecuarios podría leerse como un rompimiento con la división sexual del trabajo existente, pero en realidad significa que los hogares adaptan su discurso a las expectativas de quienes promueven los programas con el fin de obtener los beneficios. En la práctica, las mujeres siguen realizando las labores convencionales del hogar, aunque se observó un cambio de actitudes en las mujeres más jóvenes; no es posible atribuir dicho cambio a este tipo de políticas públicas o a la confluencia de otros factores, como los medios de comunicación. Las implicaciones en la "nueva ruralidad" motivan a las mujeres a salir del hogar en busca de ingresos monetarios.

Actualmente, el programa sólo apoya con un "ahorro" a la familia de estas mujeres y no logra crear empleo permanente ni cambios estructurales en la familia; entonces, los resultados del programa son marginales y poco significativos frente al problema de desigualdad que prevalece en las zonas rurales del país.
Por otra parte, debe revisarse la cobertura del programa porque el porcentaje que cubre es muy pequeño comparado con la población potencial.

Es necesario investigar cómo diseñar y operar proyectos desde una perspectiva multidimensional, aunque implique una mayor complejidad administrativa, para lograr un mayor y mejor impacto para las mujeres rurales del estado y evaluar si el empleo remunerado generado tiene viabilidad a largo plazo.

\section{Notas}

${ }^{1}$ CONEVAL (2011) en su definición de pobreza indica que son pobres quienes tienen al menos una carencia social y un ingreso menor al de la línea de bienestar.

2 Respecto a la razón obtenida con los nacimientos registrados (Cordourier-Real, 2010).

${ }^{3}$ La violencia total en la pareja actual en los últimos 12 meses de la toma de datos en 2008 (INSP, 2008).

${ }^{4}$ La exclusión social es un fenómeno multidimensional, ya sea por aislamiento físico o social de los territorios rurales y de sus habitantes, por las estructuras debilitadas del sector primario, por la carencia de empleo, por la evolución negativa de los servicios sociales o por la desestructuración de las redes familiares, entre otros muchos factores que impiden que los individuos participen plenamente de la sociedad (Cabero, 2011: 15).

5 En México, en 2009 las mujeres rurales dedicaban el $44.1 \%$ de su tiempo semanal al trabajo en el hogar, en contraste con sus pares masculinos que dedicaban menos del 9\% (INMUJERES, 2010: 6).

${ }^{6}$ Las políticas de equidad de género son estrategias de intervención pública que se aplican para corregir los desequilibrios que existen entre las personas en razón de su sexo, es decir, las desigualdades de género. Se trata de acciones de justicia deliberada que buscan compensar las posiciones de desventaja que se dan por la discriminación de género que afecta a las mujeres (Incháustegui, 2007).

7 Propietarias de unidades de producción para el desarrollo de las actividades agropecuarias o forestales manejaron terrenos, predios o parcelas, con excepción 
de las que reportaron exclusivamente vivero o invernadero (INEGI, 2007a).

${ }^{8}$ Diario Oficial de la Federación (2010), "Reglas de operación del Programa de la Mujer en el Sector Agrario (PROMUSAG)", cuarta sección, 24 de diciembre.

${ }^{9}$ El número de productoras fue de alrededor de 20000 (INEGI, 2008a).

10 La unidad de muestreo son los beneficiarios del PROMUSAG en el estado de Tabasco en los años 2004 y 2006. Los parámetros utilizados fueron:

$$
n=\frac{N^{*} p^{*} q}{\frac{(N-1) B^{2}}{Z_{\text {conf }}^{2}}+p^{*} q}
$$

$$
q=(1-p)
$$

N: población; p: porcentaje real de la población objetivo, 10\%; q: estimación de la proporción. Porcentaje que estima la investigación - percepción favorable al programa de 90\%-; Z: valor asentado en las tablas estadísticas de la distribución normal estándar correspondiente a un nivel de confianza requerido. Nivel de confianza: 90\% — valor en tablas 1645-, B: error máximo aceptable — precisión del estimador-. Máxima diferencia aceptable entre el valor real p, y su estimación q, proveniente de la investigación, 0.05.

${ }^{11}$ No se aplicaron 18 cuestionarios ya que no fueron localizadas las beneficiarias en los hogares señalados en las fichas de registro proporcionadas por el programa.

${ }^{12}$ El ingreso total del hogar está conformado por: ingresos por trabajo, otros ingresos e ingresos imputados de la vivienda.

${ }^{13}$ Se debe señalar que sólo un tercio de las encuestadas en ambos años manifestó que obtuvo ingresos por trabajo.

${ }^{14}$ Para la línea de bienestar y la línea de bienestar mínima de 2008 se tomó un promedio de los meses de marzo, abril y mayo de 2008: un ingreso per cápita de 179.5 pesos y de 596.7 pesos respectivamente (CONEVAL, 2011).

${ }^{15}$ En los grupos del año 2006 se produjo un efecto de "truncamiento" porque la investigación se realizó sobre los dos años posteriores.

\section{Referencias bibliográficas}

Appendini, Kirsten y Marcelo de Luca (2006), Género $y$ trabajo. Estrategias rurales en el nuevo contexto agrícola mexicano, Roma: FAO.

Appendini, Kirsten y Gabriela Torres Mazuera (2008), "Perspectivas multidisciplinarias de una realidad fragmentada", en Kirste Appendini y Gabriela Torres Mazuera (eds.), ¿Ruralidad sin agricultura?: perspectivas multidisciplinarias de una realidad fragmentada, México: El Colegio de México-Centro de Estudios Económicos.

Arizpe, Lourdes y Carlota Botey (1986), "Las políticas de desarrollo agrario y su impacto sobre la mujer campesina en México", en C. D. Deere y M. León (eds.), La mujer y la política agraria en América latina, Bogotá: Presencia.

Benería, Lourdes y Gita Sen (1981), "Accumulation, Reproduction, and Women's Role in Economic Development”, en Signs, vol. 7, núm. 2, pp. 279-298.

Bourdieu, Pierre y J.D. Loïc Wacquant (1995), Respuestas. Por una antropología reflexiva, México: Grijalbo.

Cabero Diéguez, Valentín et al. (2011), "Pobreza y exclusión social en el ámbito rural", en Guía de recomendaciones y líneas de actuación en inclusión social, Madrid: Fundación Luis Vives.

Carton de Grammont, Hubert (2004), "La nueva ruralidad en América Latina", en Revista Mexicana de Sociología, núm. especial 65 aniversario, México: Instituto de Investigaciones Sociales-UNAM, pp. 279-300.

Cañada Melecio, Rocío y Emma Zapata-Martelo (2005), "Gestores e innovadoras: las productoras de nopal verdulero", en Emma Zapata-Martelo (ed.), Tejiendo esperanzas. Los proyectos de mujeres rurales, México: Instituto de la Mujer Guanajuatense/Colegio de Postgraduados/GIMTRAP/INDESOL.

Consejo Nacional de Población (CONAPO) (2006), Índices de marginación 2005, México: CONAPO.

Consejo Nacional para Prevenir la Discriminación (CONAPRED) (2011), Encuesta nacional sobre discriminación en México. Resultados generales 2010, México: CONAPRED. 
Consejo Nacional de Evaluación de la Política de Desarrollo Social (CONEVAL) (2011), Metodología para la medición multidimensional de la pobreza en México, México: CONEVAL.

Cordourier-Real, Gabriela (2010), Informe sobre desarrollo humano de los pueblos indígenas en México. El reto de la desigualdad de oportunidades, México: PNUD.

Dixon, Ruth B. (1982), "Women in Agriculture: Counting the Labor Force in Developing Countries", en Population and Development Review, vol. 8, núm. 3, pp. 539-566.

Galmiche-Tejeda, Ángel y Janet Townsend (2006), "Sustainable Development and Gender Hierarchies: Extension for Semi-Subsistence”, en Gender, Technology and Development, vol. 10, núm.1, pp. 101-126.

García Vázquez, Nidia (2010), "Sin masa y sin crítica. La legislación y las políticas públicas de equidad de género en Jalisco", en A. Arellano Ríos (ed.), Del discurso a la práctica: cuatro estudios acerca de la administración pública en Jalisco, Jalisco: El Colegio de Jalisco.

Gestión Social y Cooperación (GESOC) (2012), Índice de desempeño de los programas públicos federales, México: INDEP.

INEGI (2008a), Censo agropecuario 2007, VIII Censo agrícola, ganadero y forestal, Aguascalientes: INEGI.

INEGI (2008b), Censo agropecuario 2007, IX Censo ejidal, Aguascalientes: INEGI.

INEGI (2010), Encuesta nacional sobre uso del tiempo 2009. ENUT, Aguascalientes: INEGI.

INEGI (201la), Censo de población y vivienda 2010, Aguascalientes: INEGI.

INEGI (20llb), Encuesta nacional de ocupación y empleo. Aguascalientes: INEGI.

INEGI (20llc), Encuesta nacional de ocupación y empleo. Consulta interactiva de datos. Tercer trimestre, Aguascalientes: INEGI.

Instituto Nacional de las Mujeres (INMUJERES) (2008), Programa Nacional para la Igualdad entre Mujeres y Hombres 2008-2012. PROIGUALDAD, México: INMUJERES.

Instituto Nacional de las Mujeres (INMUJERES) (2010), Las desigualdades de género vistas a través del estudio del uso del tiempo. Resultados de la encuesta nacional sobre uso del tiempo 2009, México: INMUJERES.

Instituto Nacional de Salud Pública (INSP) (2008), Encuesta de salud y derechos de las mujeres indígenas, México: INSP.

Jenson, Jane (2009), "Lost in translation: The social investment perspective and gender equality", en Social Politics: International Studies in Gender, State o Society, vol.16, núm. 4, pp. 446-483.

Kabeer, Naila (1998), Realidades trastocadas. Las jerarquías de género en el pensamiento del desarrollo, México: Paidós.

Lagarde, Marcela (1990), Los cautiverios de las mujeres: madresesposas, monjas, putas, presas y locas, México: UNAM-Programa Universitario de Estudios de Género.

Lagarde, Marcela (1996), Género y feminismo. Desarrollo humano y democracia, Madrid: Horas y Horas.

Lugones, María (2008), "Colonialidad y género", en Tabula Rasa, vol. 9, pp. 73-101.

Maeda-Machang'u, Amy Demise et al. (2000), "Gender Roles, Local Knowledge, Food Security and Biodiversity in Different Livestock Production Systems in Tanzania”, en A.S. Kauzeni (ed.), Gender, Biodiversity and Local Knowledge Systems (LinKS) to Strengthen Agricultural and Rural Development, Roma: FAO.

Martínez Corona, Beatriz (2005), "Mujeres de núcleos agrarios, liderazgo y proyectos productivos", en Emma Zapata-Martelo y J. López-Zavala (eds.), La integración económica de las mujeres rurales un enfoque de género, México: Secretaría de la Reforma Agraria.

Merino, Mauricio (2009), "Los programas de subsidios al campo. Las razones y las sinrazones de una política mal diseñada", en Documentos de Trabajo de Administración Pública, núm. 229. México: CIDE.

Mink, Stephen D. (1993), Poverty, Population, and the Environment, World Bank Discussion Paper núm. 189, Washington: The World Bank.

Molyneux, Maxine (2006), "Mothers at the Service of the New Poverty Agenda: Progresa/Oportunidades, Mexico's Conditional Transfer Programme", en Social Policy \& Administration, vol. 40, núm. 4, p. 425. 
Moser, Caroline (1989), "Gender Planning in the Third World: Meeting Practical and Strategic Gender Needs", en World Development, vol. 17, núm. 11, pp. 1799-1825.

Olivera Bustamante, Mercedes (2010), "Incidencia de la crisis en la situación y condición de género de las mujeres rurales", en B. Rubio (ed.), El impacto de la crisis alimentaria en las mujeres rurales de bajos ingresos en México 2008-2009, México: Instituto de Desarrollo Social-SEDESOL.

Pérez Fernández del Castillo, Germán et al. (2004), "Empoderamiento, individuación y estrategias para salir de la pobreza: comentarios sobre la encuesta Lo que dicen los pobres", en Cuadernos de Desarrollo Humano, núm. 19. México: Secretaría de Desarrollo Social.

Portocarrero, Patricia y Patricia Ruiz Bravo (1990), Mujeres y desarrollo. Recorridos y propuestas, Madrid: IEPALA.

Reij, Chris y Ann Waters-Bayer (eds.) (2001), Farmer Innovation in Africa: A Source of Inspiration for Agricultural Development, Londres: Earthscan.

Rota, Antonio (2010), Livestock. Thematic papers. Tools for Project Design, Roma: IFAD.

Secretaría de la Reforma Agraria (SRA) (2008), Evaluación externa de impacto 2008 del Programa de la Mujer en el Sector Agrario (PROMUSAG 20052006), México: SRA/Universidad Autónoma de Chapingo.
Secretaría de la Reforma Agraria (SRA) (2011), Evaluación complementaria de los programas de fomento de la Secretaría de la Reforma Agraria. Programa de la Mujer en el Sector Agrario (PROMUSAG), México: FAO-SRA.

Tepichin, Ana María (2005), Equidad de género y pobreza. Autonomía en beneficiarias del Programa Oportunidades. Estudio de caso. Instituto Nacional de Desarrollo SocialSEDESOL, México: Luna Quintana.

Tepichin, Ana María (2010), "Política pública, mujeres y género”, en Ana María Tepichin, Karine Tinat y Luz Elena Gutiérrez de Velasco (eds.), Relaciones de género, México: El Colegio de México.

Tepichin, Ana María (2011), "Desigualdades de género y pobreza femenina”, en Ana María Tepichin (ed.), Género en contextos de pobreza, México: El Colegio de México.

Torres, Cristina (1989), "El trabajo doméstico y las amas de casa”, en Sociológica, vol. 4, núm 10.

Tuñón Pablos, Esperanza, Rolando Tinoco Ojanguren y Armando Hernández de la Cruz (2007), “Genero y microfinanciación: Evaluación de un programa de microfinanciación para mujeres en el estado de Tabasco", en Revista de Estudios de Género La Ventana, vol. 26, núm. 3, pp. 41-69.

Zapata-Martelo, Emma (2005), "Cambios en el sector agropecuario y los proyectos de las mujeres rurales", en Emma Zapata-Martelo y J. López-Zavala (eds.), La integración económica de las mujeres rurales un enfoque de género, México: Secretaría de la Reforma Agraria. 
Cuadro 1. Características socioeconómicas de las beneficiarias del PROMUSAG en Tabasco en 2004 y 2006

\begin{tabular}{|c|c|c|c|c|c|c|}
\hline \multirow{3}{*}{ Indicador } & \multicolumn{6}{|c|}{ Beneficiarias } \\
\hline & \multicolumn{3}{|c|}{2004} & \multicolumn{3}{|c|}{2006} \\
\hline & Valor & D.E. & n válido & Valor & D.E. & n válido \\
\hline Edad promedio & 41 & 12 & 75 & 41 & 13 & 76 \\
\hline $\begin{array}{l}\text { Promedio de integrantes del } \\
\text { hogar }\end{array}$ & 5 & 2 & 75 & 5 & 2 & 76 \\
\hline Años de escolaridad & 6 & 3 & 69 & 7 & 4 & 70 \\
\hline $\begin{array}{l}\text { Porcentaje que sabe leer y } \\
\text { escribir }\end{array}$ & $86.0 \%$ & & 74 & $82.0 \%$ & & 76 \\
\hline $\begin{array}{l}\text { Porcentaje que sabe hacer } \\
\text { cuentas }\end{array}$ & $82.0 \%$ & & 74 & $75.0 \%$ & & 76 \\
\hline $\begin{array}{l}\text { Porcentaje de hogares con } \\
\text { jefatura femenina }\end{array}$ & $73.0 \%$ & & 75 & $80.0 \%$ & & 75 \\
\hline $\begin{array}{l}\text { Porcentaje que señaló estar } \\
\text { casada y en unión libre }\end{array}$ & $74.7 \%$ & & 75 & $86.8 \%$ & & 76 \\
\hline $\begin{array}{l}\text { Ingreso promedio mensual } \\
\text { per cápita del hogar (pesos } \\
\text { corrientes) }\end{array}$ & $\$ 1000$ & 821 & 74 & $\$ 1063$ & 810 & 76 \\
\hline $\begin{array}{l}\text { Promedio de horas diarias } \\
\text { dedicadas a actividades del } \\
\text { hogar }\end{array}$ & 10.7 & 4.8 & 72 & 11 & 4 & 76 \\
\hline $\begin{array}{l}\text { Porcentaje de mujeres que dijo } \\
\text { ser ejidataria }\end{array}$ & $20.4 \%$ & & 49 & $15.3 \%$ & & 59 \\
\hline
\end{tabular}

Fuente: elaboración propia con base en la encuesta a beneficiarias 2004 y 2006 en Tabasco.

El tamaño de la muestra es: n2004=75, n2005=76; N2004=449, N2006=1009. El levantamiento de la información fue realizado de octubre de 2007 a abril de 2008.

D.E.: desviación estándar. 


\section{Cuadro 2. Características de la operación del proyecto según las beneficiarias del PROMUSAG en Tabasco en 2004 y 2006}

\begin{tabular}{|c|c|c|c|c|}
\hline \multirow{3}{*}{ Variables } & \multicolumn{4}{|c|}{ Beneficiarias } \\
\hline & \multicolumn{2}{|c|}{2004} & \multicolumn{2}{|c|}{2006} \\
\hline & Porcentaje & n válido & Porcentaje & n válido \\
\hline Eligió el proyecto porque era más fácil & 64.4 & 59 & 42.9 & 49 \\
\hline Empezó a trabajar en el proyecto & 96.6 & 58 & 95.3 & 64 \\
\hline Trabajó el proyecto de manera individual & 36.8 & 68 & 31.9 & 72 \\
\hline Trabajó el proyecto de manera conjunta & 45.6 & 68 & 56.9 & 72 \\
\hline $\begin{array}{l}\text { Trabajó en el proyecto personalmente (no lo trabajó un } \\
\text { tercero o familiar) }\end{array}$ & 50.0 & 70 & 33.8 & 65 \\
\hline $\begin{array}{l}\text { Las actividades que realizó en el proyecto fueron las } \\
\text { planeadas }\end{array}$ & 79.7 & 69 & 89.6 & 67 \\
\hline $\begin{array}{l}\text { Considera que le dieron información suficiente y oportuna } \\
\text { sobre el programa }\end{array}$ & 73.2 & 71 & 65.7 & 67 \\
\hline \multicolumn{5}{|l|}{ ¿Cuántas veces recibió asesoría técnica o capacitación? } \\
\hline Una o dos veces & 42.3 & 71 & 31.5 & 73 \\
\hline Más de dos veces & * & 71 & 27.4 & 73 \\
\hline Nunca & 36.6 & 71 & 41.1 & 73 \\
\hline $\begin{array}{l}\text { Porcentaje de sobrevivencia de proyectos de más de dos } \\
\text { años }\end{array}$ & 64.5 & 62 & 69.0 & 55 \\
\hline $\begin{array}{l}\text { Consideró que la parte más importante para que el } \\
\text { proyecto se desarrolle bien es la cooperación grupal }\end{array}$ & 47.1 & 51 & 51.9 & 54 \\
\hline
\end{tabular}

Fuente: Elaboración propia con base en la encuesta a beneficiarias 2004 y 2006 en Tabasco.

El levantamiento de la información fue realizado de octubre de 2007 a abril de 2008.

* Menos de 20 observaciones. 
Cuadro 3. Percepción de las beneficiarias del PROMUSAG en Tabasco en 2004 y 2006

\begin{tabular}{|c|c|c|c|c|}
\hline \multirow{3}{*}{ Variables } & \multicolumn{4}{|c|}{ Beneficiarias } \\
\hline & \multicolumn{2}{|c|}{2004} & \multicolumn{2}{|c|}{2006} \\
\hline & Porcentaje & n válido & Porcentaje & n válido \\
\hline $\begin{array}{l}\text { Consideró que el programa ayuda a las mujeres a salir de } \\
\text { la pobreza }\end{array}$ & 84.9 & 73 & 77.5 & 71 \\
\hline Señaló que el apoyo recibido cambió sus vidas & 84.3 & 70 & 66.2 & 65 \\
\hline \multicolumn{5}{|l|}{$\begin{array}{l}\text { ¿Cuánto esperaba que la ayudara a vivir mejor el apoyo } \\
\text { que recibió? }\end{array}$} \\
\hline Mucho & 34.8 & 69 & 37.9 & 66 \\
\hline Poco & 60.9 & 69 & 54.5 & 66 \\
\hline \multicolumn{5}{|l|}{$\begin{array}{l}\text { Una vez que recibió el apoyo, ¿qué tanto le sirvió para vivir } \\
\text { mejor? }\end{array}$} \\
\hline Mucho & 28.6 & 70 & * & 66 \\
\hline Poco & 57.1 & 70 & 54.5 & 66 \\
\hline Se sintió satisfecha con los beneficios del proyecto & 69.6 & 69 & 79.7 & 59 \\
\hline Señaló que el principal beneficio para el hogar fue el ahorro & 53.7 & 67 & 50.8 & 63 \\
\hline $\begin{array}{l}\text { Señaló que el principal problema que han tenido con el } \\
\text { proyecto es el gasto que conlleva }\end{array}$ & 38.6 & 57 & 50.0 & 48 \\
\hline
\end{tabular}

Fuente: Elaboración propia con base en la encuesta a beneficiarias 2004 y 2006 en Tabasco.

El levantamiento de la información fue realizado de octubre de 2007 a abril de 2008.

* Menos de 20 observaciones. 
Gráfico 1. Promedio de ingresos de las beneficiarias del PROMUSAG y de otros ingresos del hogar: 2004, 2006 (en pesos corrientes)

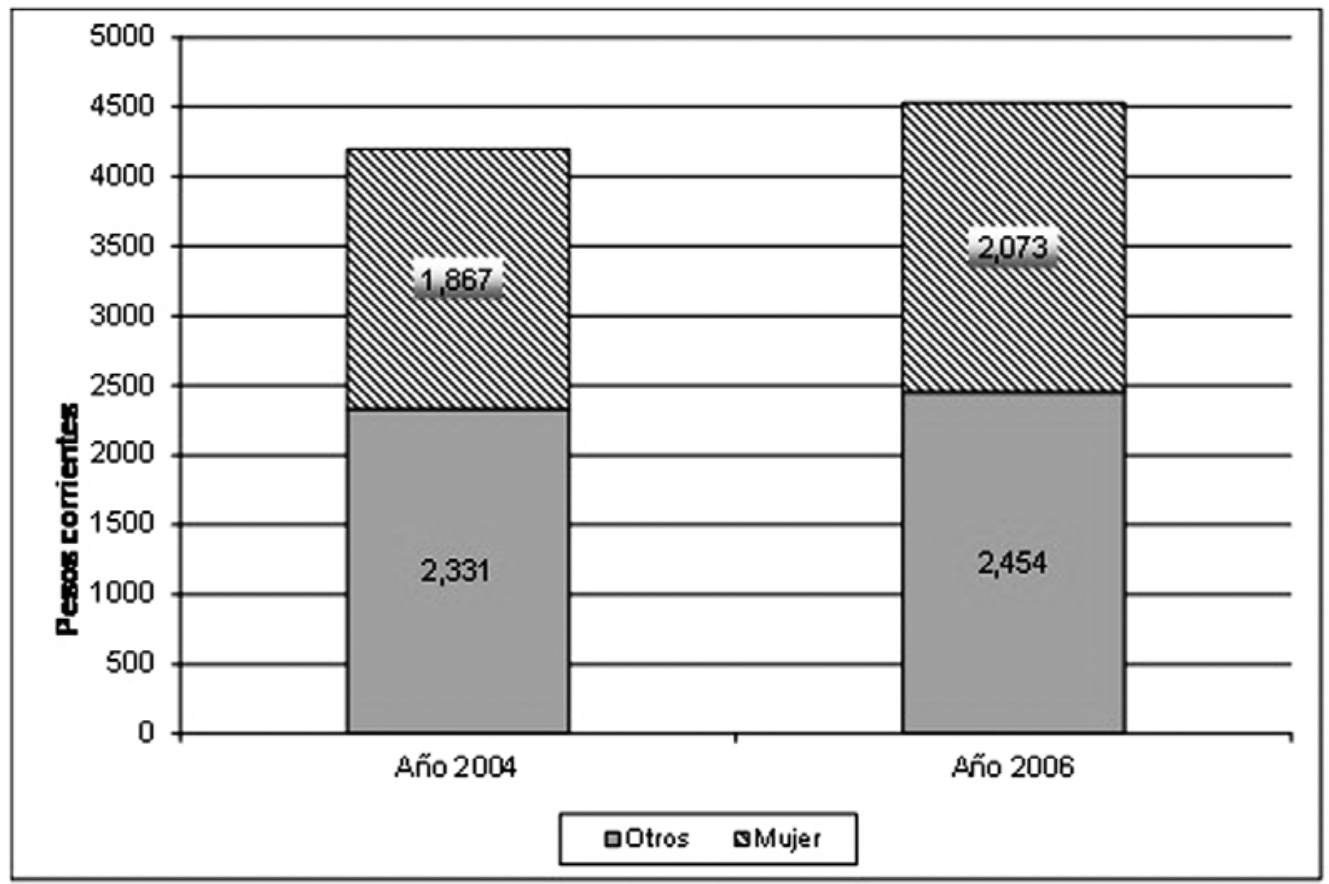

Fuente: elaboración propia con base en la encuesta a beneficiarias 2004 y 2006 en Tabasco.

Gráfico 2. Cumplimiento de expectativas de las beneficiarias que creyeron que el PROMUSAG les ayudaría a salir de la pobreza, 2004, 2006 (en \%)

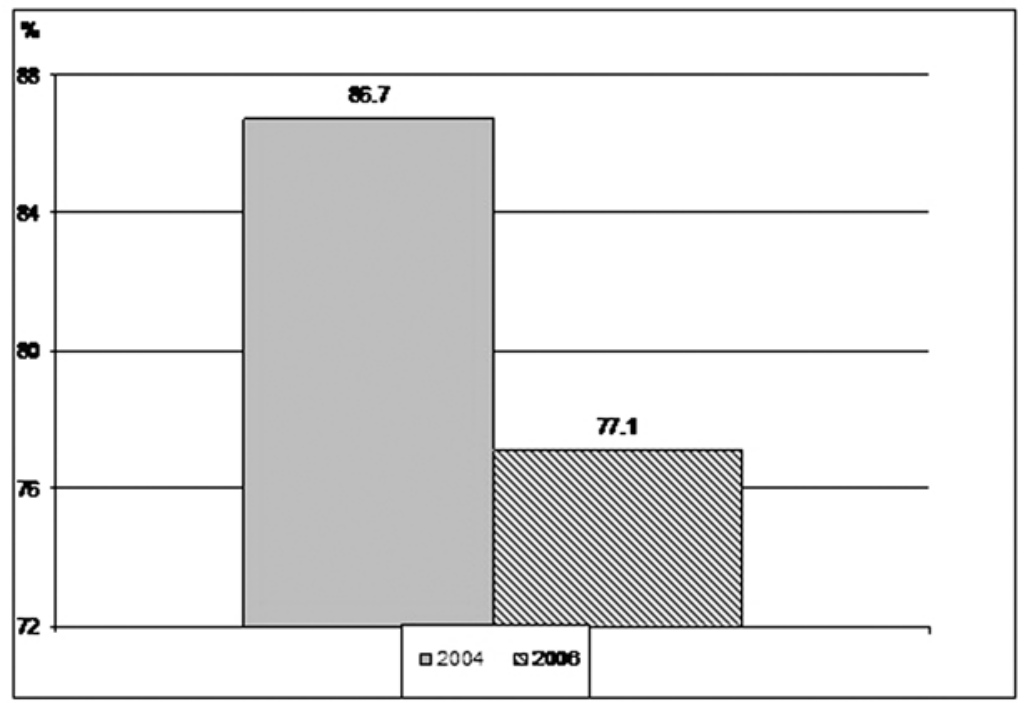

Fuente: elaboración propia con base en la encuesta a beneficiarias 2004 y 2006 en Tabasco. 